\title{
Nutrition and pregnancy: an update
}

\begin{abstract}
The period of pregnancy, is a period when multiple physical and body changes are taking place for the woman. The nutrition of the woman during, and before the pregnancy, is a decisive factor for the health of the woman and of the fetus health as well. According to the American Dietetic Association, females that are during fertile age, have to follow a balanced and healthy diet. This implies the consumption of a variety of food according to the food pyramid, supplementary vitamins and minerals, and the safe processing and preparation of the food (journal of the American Dietetic Association). The healthy nutrition contributes to the desired increase in weight, and the successful outcome of the pregnancy. Nevertheless, it constitutes a factor that can affect the development of the foetus, the birth weight of the newborn, the development of disorders at the foetus, as well as the development of chronic problems in later life. The needs of the foetus in nutrients and energy, vary depending on the stage of growth, thus the diet of the mother should be adapted to that factor. Thus, the nutrition of the mother must be adaptive to these needs. Nutrients are the substances that are absorbed through the food and are used for the body as an energy source. 1 There are six categories of nutrients, proteins, carbohydrates, fats, inorganic components, vitamins and water.
\end{abstract}

Volume 5 Issue $3-2019$

\author{
Anastasia Tzelali, Stamatios Petousis, \\ Chrysoula Margioula-Siarkou, Georgia \\ Margioula-Siarkou, Konstantinos Dinas, \\ George Mavromatidis \\ 2nd Department of Obstetrics and Gynaecology, Aristotle \\ University of Thessaloniki, Greece
}

Correspondence: Stamatios Petousis, 2nd Department of Obstetrics and Gynaecology, Aristotle University of Thessaloniki, Greece,Email petousisstamatios@gmail.com

Received: May 17, 2019| Published: June 06, 2019

\section{Introduction}

\section{Proteins}

Proteins are the most known macromolecules. They consist of amino acids, which are connected to each other with peptide bonds, forming chains. The human body uses twenty amino acids to form the proteins but it cannot form eight amino acids, thus, they have to be taken through food. These amino acids are called essential amino acids. Proteins are necessary for the formation and the regeneration of the tissue, synthesis of hormones, enzyme, antibodies, and many other functions. The maternal needs in protein increase, especially during the third trimester, nevertheless, they are necessary throughout the whole duration of the pregnancy. ${ }^{2}$

The sources of the proteins differ according to their nutrition value. Proteins of animal origin, such as meat, fish, poultry, eggs and diary products, are considered to be of higher protein quality due to the fact that they contain all the necessary amino acids. In the contrast, plantderived proteins, are considered to be of lower quality, because they do not contain all the amino acids. Low intake of protein during the pregnancy is associated with low birth weight and increased maternal morbidity, while high protein intake, has negative effects on the birth weight and it should be avoided. ${ }^{3}$

\section{Carbohydrates}

Carbohydrates provide the highest nutrition energy source. The carbohydrates that are metabolised and absorbed in the small intestine, are divided in simple and complex, depending on the number of sugars they have. The carbohydrates that cannot be broken down in the small intestine, and pass to the large intestine, are called fibers. Food that is rich in carbohydrates is cereal, pasta, bread, legumes, rice, potatoes, fruit, vegetables, honey etc. The consumption of the carbohydrates is very important during pregnancy, because they have glucose for the foetus and the woman. The consumption of fibers is necessary for the proper function of the intestine especially during pregnancy, since the augmented levels of progesterone, affect the ton (EINAI AYTO $\Sigma \Omega \Sigma T O$ ?) of the smooth muscle fibers in the gastrointestinal system. ${ }^{3}$

\section{Fats}

Fats are chemical compounds of glycerin with organic acids. They are distinguishing in animal and plant origin. The animal origin are contained in meat, fish, eggs, milk, butter and cheese, while the plant origin are contained in olive oil, corn oil, sunflower oil and seed oil. Fats are used to produce and store energy. They are an appropriate "raw material" for energy production, since each gram of fat yields $9 \mathrm{kcal}$, when carbohydrates and proteins yield $4 \mathrm{kcal}$, respectively. Polyunsaturated fat acids contribute to the normal development of the fetus' brain. Insufficiency of these, may affect the neonatal development.

\section{Inorganic components}

Inorganic components are found in the human body either alone, or in the form of compounds. They are necessary because they regulate the acid-base balance of the body and participate in important metabolic pathways. The most important inorganic ingredients that pregnant women need to take in adequate amounts are iron, calcium, magnesium, zinc, phosphorus, potassium, sodium, and chlorine.

\section{Vitamins}

Vitamins are substances that are found in food in small amounts. Their intake is necessary, due to the fact that the human body cannot compose them. Their antioxidant action, and their necessary presence to convert macromolecules (carbohydrates, proteins, fats) into energy, make them valuable to pregnancy. Late intrauterine embryonic development, placental abruption and maternal anemia are associated with decreased vitamin A consumption, while overconsumption is associated with teratogenic effects. Food rich in vitamin A are cheese, egg and milk. ${ }^{4}$ Vitamin D preserves calcium levels in the blood at 
normal limits, increasing its absorption from the intestine, and it also participates in bone synthesis. Nevertheless, excessive consumption can be toxic. Food rich in vitamin D I $\Sigma$ meat, fatty fish and egg. ${ }^{4}$

\section{Water}

Water is the main constituent of the human body. During pregnancy, the amount of body fluids increases by $50 \%$. Therefore, there is an increased need for fluid consumption. Consuming water is good between and not during the meals. ${ }^{5}$

\section{Special dietary considerations during each pregnancy trimester}

\section{First trimester of pregnancy}

The first trimester, is the stage of organogenesis of the foetus. The foetuses requirements in proteins (which are the structural component of the body) and carbohydrates (energy source) are increased. The pregnant woman starts to take folic acid supplements, a dose of at least 400 mgr per day. The folic acid can reduce the risk of nervous tube abnormalities and other genetic anomalies, especially for the high risk pregnancies. Vegetarians must also take supplement vitamins B12. The intake of vitamin A above the limit of 10.000 IU per day, during the first stages of the pregnancy can cause genetic anomalies.

During the first trimester, often there is intense nausea and vomiting tendency for the pregnant woman, mainly due to the hormonal changes that occur in her body. Thus, it is recommended that the nutrition of the pregnant, should not include food that irritate the stomach, and many and small meals are recommended. Furthermore, the consumption of food with high concentration in carbohydrates and potassium, and low concentration in fat, is recommended. In conclusion, the simultaneously consumption of liquid and solid food has to be avoided, half an hour period between these two has to occur.

\section{Second trimester of pregnancy}

During the second trimester of pregnancy, the nutrition needs are increased, as it is the stage of the doubling of the organs and tissue of the foetus. In this stage, the first nutrients that will help the pregnant and the foetus are highly biological value proteins, because as a structural component of the body, they will do this work. Meat, poultry, fish, legume and dried nuts, are important sources of protein. During the second trimester of the pregnancy, the pregnant woman is necessary to start taking iron and calcium. Food rich in iron is the red meat, fish, poultry and dried fruit. The supplement of iron has to be at least $30 \mathrm{mgr}$ per day. The daily intake of calcium has to be at least $1000 \mathrm{mgr}$. Food rich in calcium is dairy products, such as milk, cheese and yogurt. ${ }^{6}$

\section{Third trimester of pregnancy}

During the third trimester of the pregnancy, an excessive increase of the body of the foetus occurs. The nutrition of the pregnant woman in this stage of the pregnancy is highly important and it has the primary role in the development of the foetus. The increased nutrition needs of the woman and the embryo, in qualitative and quantitative terms, must be met with meals that include high calorie food. In the last months of the pregnancy, there is often a constipation problem, mainly due to the increase of the progesterone in the body. To overcome this problem, it is recommended food rich in fiber to be consumed. Such food is fruit, vegetables, legumes, and whole grain cereal. ${ }^{7}$

\section{Conclusion}

From all the above, it is understandable how important is the balanced nutrition for the pregnant woman. Fruit and vegetables has to be consumed on a daily bases, to cover the need in nutrient and fiber. Food with rich sources in protein, iron and magnesium is, meat, fish, poultry, legume and dried nuts. Carbohydrates are rich in cereals, such as bread, pasta and rice. Calcium is in diary products, however, women that are lactose intolerant can replace milk with dairy products that have lower concentration in lactose, such as cheese and yogurt. As concerns the hydration of the body, it is achieved with the daily consumption of 8-10 glasses of fluids, such as water, juice, milk etc. ${ }^{8-}$ ${ }^{10}$ Pregnancy is a very important period in the life of a woman, in the duration off, her nutrition condition is a key for her health and the baby's health, as well. The correct nutrition is a valuable helper in the treatment of physical and psychological challenges which the woman will face during her pregnancy. The food quality improvement is the key to face the increased needs and not to double the amount of food. Right nutrition helps the mother for a healthy pregnancy and an easier delivery while excessive weight gain may cause complications during the pregnancy and the labor as well.

\section{Acknowledgments}

None.

\section{Conflicts of interest}

The author declares there are no conflicts of interest.

\section{References}

1. Tzika A. Nutrition during pregnancy. Athens; 2009.

2. Duggleby SL, Jackson AA. Protein, amino acid and nitrogen metabolism during pregnancy: how might the mother meet the needs of her fetus? Curr Opin Clin Nutr Metab Care. 2002;5(5):503-509.

3. Food and Nutrition Guidelines for Healthy Pregnant and Breastfeeding Women: A background paper. Ministry of Health. New Zealand; 2006.

4. Vrentzou L, Pantalaki A, Healthy diet education in pregnancy. Creta; 2007.

5. Eisenberg A, Murkoff H, Hathaway SE. What to expect when you are pregnant. Dioptra, Athens; 2002.

6. Williamson CS. Nutrition in Pregnancy. British Nutrition Foundation. Nutrition Bulletin. 2006;31:28.

7. James A Greenberg, Stacey J Bell, Yong Guan, et al. Folic Acid Supplementation and Pregnancy: More Than Just Neural Tube Defect Prevention. Rev Obstet Gynecol. 2011;4(2):52-59.

8. Position of the American Dietetic Association: Nutrition and lifestyle for a healthy pregnancy outcome. 2002.

9. Olsen SF, Secher NJ. Low consumption of seafood in early pregnancy as a risk factor for pre-term delivery: prospective cohort study. BMJ. 2002;324(7335):447.

10. Zampelas A. Nutrition in life stages, Paschalides, Athens; 2003. p. 10-62. 